\title{
New perceptions on the $S$ Doradus phenomenon and the micro variations of five Luminous Blue Variables (LBVs) ${ }^{\star}$
}

\author{
A.M. van Genderen ${ }^{1}$, M. de Groot ${ }^{2}$, and C. Sterken ${ }^{3, \star \star}$ \\ 1 Leiden Observatory, Postbus 9513, 2300 RA Leiden, The Netherlands \\ 2 Armagh Observatory, College Hill, Armagh BT61 9DG, Northern Ireland \\ 3 University of Brussels (VUB), Pleinlaan 2, 1050 Brussels, Belgium
}

Received July 8; accepted December 17, 1996

\begin{abstract}
We investigated the photometric histories of the LBVs HR Car in the Galaxy, R 127, R 110 and R 71 in the LMC, and R 40 in the SMC by collecting all available photometry. All these objects have been reasonably well observed over the last few decades and a number of "S Dor (SD) phases" (episodes of enhanced light) is well documented. Time-scales lie between 1.4 and 25 y. During the SD cycles two different types of micro-variations (amplitudes $\sim 0$. 2 ) are present: one near the minima (time-scale from 2 to 6 weeks; colours generally blue in the maxima and red in the minima) and the other one near the maxima (time-scale $\sim 100 \mathrm{~d}$; colours generally red in the maxima and blue in the minima). Halfway the ascending and descending branches of the SD cycles the stars switch rather abruptly from one type to the other. We argue that the two types of SD phases are probably caused by different instability mechanisms.
\end{abstract}

Key words: technique: photometric - stars: individual HR Car = HD 90177, R $127=$ HDE 269858f, R $40=$ HD 6884, R $71=$ HDE 269006, R $110=$ HDE $269662-$ stars: variables — stars: supergiants — stars: oscillations

\section{Introduction}

Recently, we have investigated the photometric history of the hot S Dor type stars, or Luminous Blue Variables (LBVs) AG Car, S Dor and $\eta$ Car comprising over a century of photometric observations (van Genderen et al. 1996, hereafter called Paper I; de Groot et al. 1996). We proposed that the S Dor (SD) activity is caused by two types of SD phases: the very long-term (VLT-SD) and the

Send offprint requests to: A.M. van Genderen

* Based, in part, on observations obtained at the European Southern Observatory at La Silla, Chile.

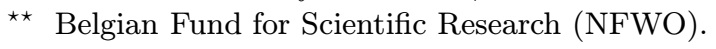

normal SD phases. The latter are superimposed on the former. Since in the visual maxima the mass-loss rate is generally not much higher than during the minima (e.g. de Koter et al. 1996), one should not call the light maxima "SD eruptions", but rather "SD phases". In the present paper we investigate the photometric histories of five other LBVs, although the time intervals over which the stars were well monitored are generally much shorter than a century. We attempt to categorize the various long- and short-term light and colour variations.

\section{Remarks to Table 1:}

1 The 700 estimates were averaged by her about each $200 \mathrm{~d}$ and given in the international magnitude system.

${ }^{2}$ Walraven $V$ transformed into $V_{\mathrm{J}}$ of the $U B V$ system.

3 Shown as a smooth curve by Hutsemékers \& van Drom (1991).

4 Association Française des Observateurs d'Etoiles Variables. Scatter amounting to \pm 0.1 in the maxima and $\sim 0.3$ in the minima, but the observations are represented by a smooth curve.

5 Observations made by the observers of the Long-Term Photometry of Variables project organized by Sterken (1983) and published by Manfroid et al. (1991, 1994) and Sterken et al. $(1993,1995)$. Note that $y \equiv V_{\mathrm{J}}$.

${ }^{6}$ For a first discussion (of part) of these observations see Spoon et al. (1994).

${ }^{7}$ Observations made by the Geneva observers. Geneva $V$ close to $V_{\mathrm{J}}$ of the $U B V$ system.

8 The following data were rejected because of their unexplainably large deviations JD 244 - 5925.868, 7405.914, 7468.730, 7477.621, 7480.617, 7777.877, 7792.840, 7825.682, 7836.741, 8099.930 and 8255.616. It is possible that the observers erroneously observed the nearby star R $128=\mathrm{HDE} 269859$, or that the close optical companion HDE 269858p ( $\left.2^{\prime \prime}\right)$, also a supergiant in the LMC, is variable as well.

9 Observations in various photometric systems, if necessary transformed to $V_{\mathrm{J}}$, based on the work by Stahl et al. (1983), de Groot (quoted by Stahl et al. 1983) and van Genderen (1970). 
Table 1. List of data sets and the type of photometry. For HR Car bracketed abbreviations are used in Fig. 1

\begin{tabular}{|c|c|c|c|}
\hline Star & Type & Reference/Group & Remarks \\
\hline \multirow[t]{11}{*}{$\overline{\mathrm{HR}} \mathrm{Car}$} & $\mathrm{pg}$ & Hoffleit (1940) (H pg) & 1 \\
\hline & $V B L U W$ & Graham (1968) $\left(\mathrm{Gh} V_{\mathrm{J}}\right)$ & 2 \\
\hline & $U B V$ & Bond \& Landolt (1970) (BL $\left.V_{\mathrm{J}}\right)$ & \\
\hline & $U B V$ & Wisse \& Wisse (1971) (WW $\left.V_{\mathrm{J}}\right)$ & \\
\hline & $V B L U W$ & van Genderen et al. (1990) (vG et al. $V_{\mathrm{J}}$ ) & 2 \\
\hline & $V B L U W$ & unpublished, made in 1990 ( $\mathrm{vG}$ et al. $V_{\mathrm{J}}$ ) & 2 \\
\hline & vis & Bateson (1987-1990) (Ba mv) & 3 \\
\hline & vis & $\operatorname{AFOEV}(\mathrm{A} \mathrm{mv})$ & 4 \\
\hline & uvby & LTPV $\left(\mathrm{LTPV} V_{\mathrm{J}}\right)$ & 5,6 \\
\hline & $u v b y$ & Kilkenny et al. (1985) (K et al. $V_{\mathrm{J}}$ ) & 6 \\
\hline & Geneva & $\left(G, V_{\mathrm{J}}\right)$ & 6,7 \\
\hline \multirow[t]{3}{*}{$\mathrm{R} 127$} & $u v b y$ & LTPV & 5,8 \\
\hline & $V B L U W$ & unpublished, made in $1983,1986-1990$ & 2 \\
\hline & various & & 9 \\
\hline \multirow[t]{3}{*}{$\mathrm{R} 40$} & $u v b y$ & LTPV & 5,10 \\
\hline & $V B L U W$ & unpublished, made in 1987-1989 & 2 \\
\hline & various & & 11 \\
\hline \multirow[t]{4}{*}{$\mathrm{R} 71$} & $V B L U W$ & van Genderen (1979) & 2 \\
\hline & $V B L U W$ & van Genderen et al. $(1985,1988)$ & 2,6 \\
\hline & $V B L U W$ & unpublished, made in 1988 and 1989 & 2 \\
\hline & $u v b y$ & LTPV & 5,6 \\
\hline \multirow[t]{4}{*}{$\mathrm{R} 110$} & $V B L U W$ & Walraven \& Walraven (1977) & 2,12 \\
\hline & various & & 13 \\
\hline & $u v b y$ & LTPV & 5 \\
\hline & $V B L U W$ & unpublished, made in 1989-1991 & 2 \\
\hline
\end{tabular}

10 Observations in the $u$ passband rejected because of their peculiar values: JD 9292.7248, 9292.6728 and 9301.5615.

11 Scattered observations collected from the literature by Szeifert et al. (1993).

12 Made in the interval 1957-1963; only the average is given by them.

13 Observations in various photometric systems, collected from the literature by Stahl et al. (1984, 1990, those of Walraven \& Walraven are mentioned separately in Remark 12), to which we added observations by Isserstedt (1975) made in the interval 1971-1973 and by van Genderen et al. (1982) made in October 1979.

\section{The observations and global light and colour curve characteristics}

The observations used for the present study are based on a large number of diverse photometric data sets, an overview of which is given Table 1. Table 2 lists the global characteristics of the light and colour curves.

\section{HR Car}

\subsection{The light curve of HR Car, 1889-1993}

In Fig. 1 a century of photometry of HR Car $=$ HD 90177, 1889-1993, is put on one scale so that a clear overview of the long-term trend is possible. The same was done in Paper I for AG Car and S Dor. Since the colour index $(B-V)_{\mathrm{J}}$ (of the $U B V$ system) of HR Car amounts to 0.85 in the minimum and to 0.95 close to the maximum, the photographic observations are corrected by 0.9 assuming that blue-sensitive plates were used. See Table 2 for the global characteristics of the light and colour curves. The smooth continuous curve in Fig. 1 is a copy of Hoffleit's (1940) light curve, corrected (brightened) by 0.9 . Dotted sections in the modern part of the light curve are not covered by observations. The abbreviations used in Fig. 1 are explained in Table 1. It appears that, on average, the visual light curves (Ba mv) and (A mv) are fainter than the $V_{\mathrm{J}}$ light curve by $0.3-0^{\mathrm{m}} \cdot 5$, but their general trends are the same as for the $V_{\mathrm{J}}$ curve. The (Ba mv) light curve shows a dip not seen in the simultaneously observed parts of the $V_{\mathrm{J}}$ curve. It seems that the dip is caused by some estimates of poor quality but, since the individual observations were not published, this cannot be checked. The continuous curve in the upper panel of Fig. 1 and 


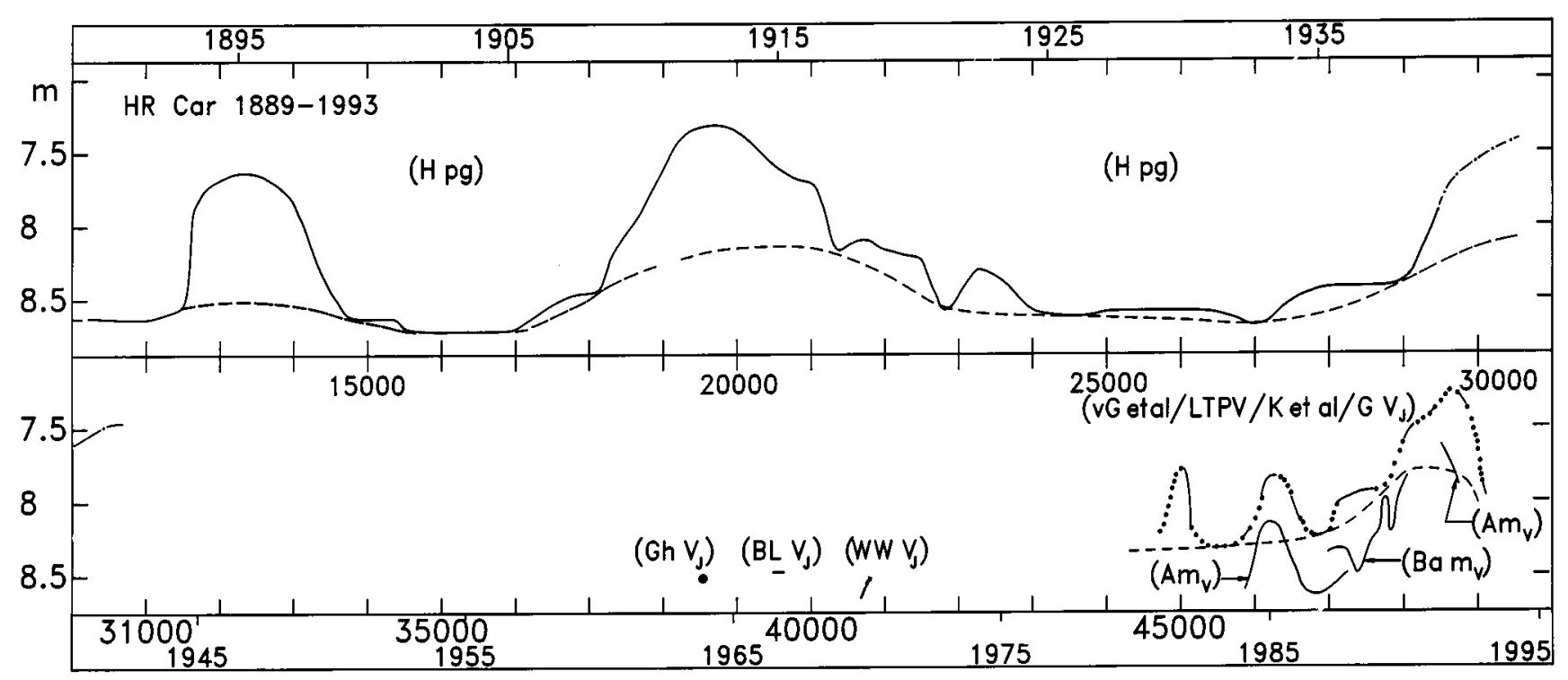

Fig. 1. The light curve of HR Car, 1889-1994 as a function of JD (minus 2440000). See the text and Table 1 for further explanation. Dates mark the beginning of the year

the small portions in the lower panel represent the wellcovered parts of the light curve. Small-amplitude features, $<0$ m. 2 with a time scale $\ll 200 \mathrm{~d}$, in the last part of the photoelectric light curve were smoothed away. They will be discussed in Sect. 3.3. The small piece of dot-dashed curve at the right of the upper panel is the extrapolation of the observed rising branch, probably the onset of a third high maximum around JD 2430000. The brightness rise after JD 2445000 is the start of another high maximum. From Fig. 1 it can be deduced that the apparent visual brightness varied over a range of $1{ }^{\mathrm{m}} 4$ (between $m_{v}=7.35$ and 8.7) during the last $107 \mathrm{y}$.

\subsection{The time scales of the $S D$ phases and the micro variations of HR Car until 1987}

Following the results of the analysis of AG Car and S Dor in Paper I, we assume that LBVs exhibit two types of SD phases with different cycle lengths, the VLT- and the normal SD phases, presumably caused by two different pulsational modes.

According to this interpretation, the VLT-SD light curve of HR Car is the lower enveloping line touching the minima and roughly represented by the broken curve in Fig. 1. All features superimposed thereon are the normal SD phases. For AG Car and S Dor an unambiguous cycle length could be established for these normal SD phases (Paper I).

In HR Car the maximum amplitude of the VLT variation is of the order of $0{ }^{\mathrm{m}} 5$, which is much smaller than for AG Car and S Dor (1-2 mag). The peak-to-peak cycle lenghts (the observed high maxima in Hoffleit's photographic light curve) lie between 20 and 50 years, which compares very well with the VLT-SD cycles of AG Car (25 y) and S Dor (35y). Normal SD phases cannot be studied in the Hoffleit (1940) data since only 200-d means were published.

The light curve after JD 2444000 is not suitable for such an analysis, either due to its short time span $(\sim 12 \mathrm{y})$ or its many time gaps. At the beginning, two normal SD phases with amplitudes $\sim 0.5$ were only partly observed; their total durations are between 2 and $2.7 \mathrm{y}$.

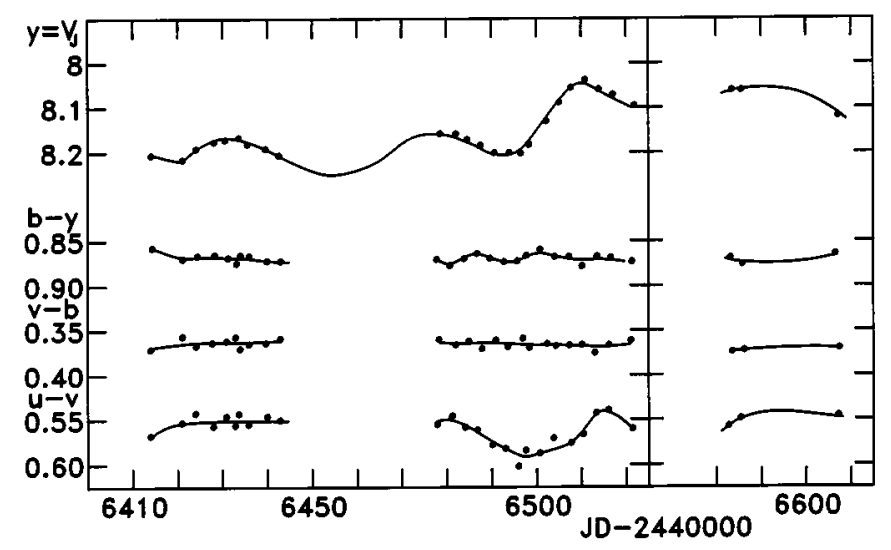

Fig. 2. Detailed light and colour curves of the $\sim 40 \mathrm{~d}$ micro variations in the hump of the descending branch of HR Car's normal SD phase (with a maximum at JD 2446250). Note that the magnitude scales for the colour curves are twice that for the light curve

The observed part of the descending branch of the lastmentioned SD phase, shown as a line segment running 
downward in Fig. 1, in reality has a small hump on which a few cycles of micro-variations are present with an average time scale of $\sim 40 \mathrm{~d}$ and amplitudes of at most 0.15 . They are shown in detail in Fig. 2 and are based on the LTPV observations. Colour variations, with the exception of $u-$ $v$, are absent. The $u-v$ colour index is blue in the maxima and red in the minima (see also Sect. 3.3). Note that the magnitude scales for the colour curves are twice those for the light curves. A shoulder appears between JD 2447000 and JD 2447600 which is the precursor of a high broad maximum discussed in Sect. 3.3.

\subsection{The fine structure and the photometric characteristics of the 1987-1994 maximum of HR Car}

Figure 3 shows the detailed light and colour curves of the high maximum of HR Car between 1987 and 1994. There is excellent agreement between the various $V_{\mathrm{J}}$ scales. The uncertainty in each data point is of the order of $\pm 0 . \mathrm{m} 01$. The $(B-V)_{\mathrm{J}}$ curve (scale at the right) is based on the transformed $V-B$ of the Walraven system. The $b-y$, $v-b$ and $u-v$ colour curves are in the instrumental system and were obtained by adding the average value of the comparison star A (= HD 93010) to the individual values of variable minus star $A$.

Note that the magnitude scales for the colours $b-y$, $(B-V)_{\mathrm{J}}, v-b$ and $u-v$ are twice those for the light curves. Individual data points were omitted in those parts of the curves where their number was too large and the scatter very low. These parts are piecewise represented by thin continuous lines. All these line pieces and scattered differential magnitudes were connected by dotted curves. The two framed sections in the $V_{\mathrm{J}}$ curve consist partly of $V B L U W$ observations. Those of the frame at the left (which is part of the "shoulder" mentioned in Sect. 3.2) were discussed in detail by van Genderen et al. (1990). Those of the second frame higher up in the light curve are shown in detail in Fig. 4.

The reddening trend during the 5 years of brightness rise after 1988, is typical for an SD phase. The range of the increase in brightness decreases towards shorter wavelengths, but is less extreme than for S Dor (Paper I) and R 127 (Sect. 4) because the variation in temperature is smaller. In the light minima of 1981 and 1987 HR Car has a temperature $T_{\text {eff }}=14000 \pm 2000 \mathrm{~K}$ (van Genderen et al. 1990), in agreement with the spectral type B4-5 determined by Shore et al. (1990). Temperatures in the minima of S Dor and R 127 are at least $20000 \mathrm{~K}$. In the light maxima the temperatures cannot be much less than, say, $8000 \mathrm{~K}$ (e.g. Wolf 1989), perhaps with the exception of R 110 (Sect. 7).

Part of the shoulder shows two simultaneous types of micro-variations: a series of $20 \mathrm{~d}$ micro-variations (blue in the maxima and red in the minima) with a light amplitude of 0.06 superimposed on $\sim 100 \mathrm{~d}$ micro-variations with amplitudes of $0{ }^{\mathrm{m}} 1-0^{\mathrm{m}} \cdot 2$ (detailed light and colour

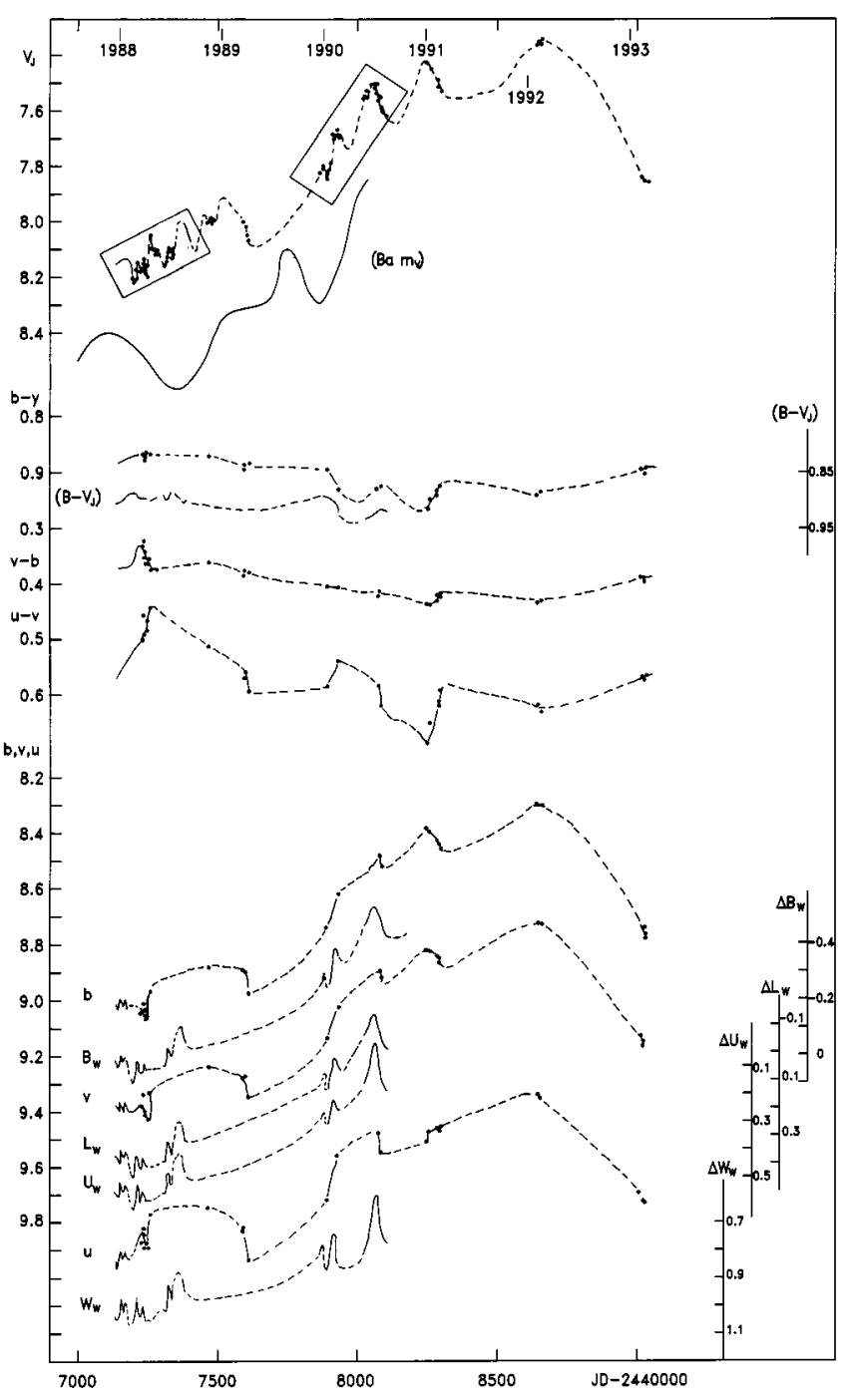

Fig. 3. The fine structure of the 1987-1994 light maximum of HR Car in $V_{\mathrm{J}}$, the colour indices $(B-V)_{\mathrm{J}}$ (scale at the right; derived from the Walraven $V-B$ ) and of the Strömgren system, and the blue and ultraviolet light curves of the Strömgren and Walraven systems sorted in order of effective wavelength (all in magnitude scale). Dates mark the beginning of the year

curves in van Genderen et al. 1990). Note that these $20 \mathrm{~d}$ micro-variations are half as short as the variations detected about two years earlier (Sect. 3.2). After about $500 \mathrm{~d}$ the $20 \mathrm{~d}$ variations disappear (with too few observations to check when and how this happened). The colour behaviour of the $100 \mathrm{~d}$ variations is red when bright and blue when faint. (It should be noted that in the lastmentioned reference they were interpreted as short-lasting SD phases and were still called "SD eruptions" but, as we proposed in Paper I, they should have been called "SD phases").

At JD 2447880, higher up in the ascending branch (see the detailed photometry in the $V B L U W$ system in Fig. 4), there is still a $20 \mathrm{~d}$ hump (time scale uncertain 


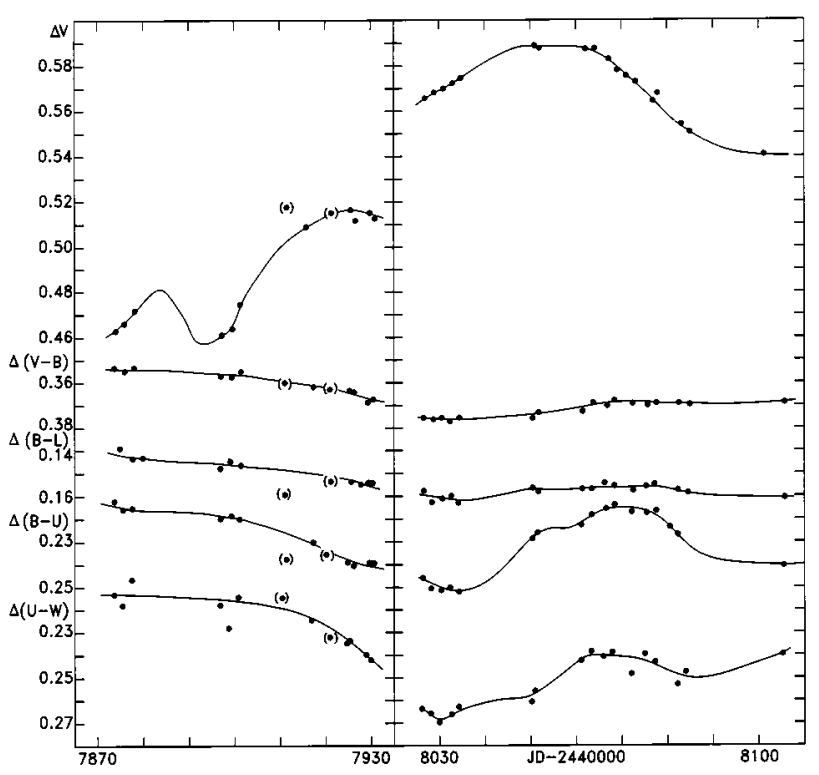

Fig. 4. The detailed light and colour variations of HR Car in the Walraven system (upper framed part of the light curve in Fig. 3) relative to the comparison star and in log intensity scale (bright and blue are up)

due to the lack of observations prior to this hump) after which these short-time scale micro-variations are definitely absent; see the smooth $\sim 100 \mathrm{~d}$ curve in the righthand panel of Fig. 4. Besides, there is now a clear tendency for the maxima in the light curve to become red. We shall see that the phase lag between light and colour curves is also, and very convincingly, present in nearly all of the $100 \mathrm{~d}$ micro-variations of R $127, \mathrm{R} 40$ and $\mathrm{R} 71$ (see further in this paper). During its maximum $\mathrm{S}$ Dor also showed micro-variations with a time scale of $\sim 100 \mathrm{~d}$ (of which half showed a mixed colour behaviour, Paper I). Sterken $(1977,1981)$ and Sterken et al. (1991) also found this kind of micro-variation (quasi-period $\sim 100 \mathrm{~d}$ ) in the maximum of the LBV HD 160529.

\section{R 127}

\subsection{The light and colour curves of $R$ 127, 1980-1994}

R $127=$ HDE 269858 f was discovered as a new S Dor variable or LBV by Stahl et al. (1983; see also Stahl \& Wolf 1986). Its spectrum at minimum light was OIafpe or, alternatively, WN9-10 (Walborn 1977, 1982). Table 2 lists the global characteristics of light and colour variations.

Figure 5 show the detailed light and colour curves comprising the time interval 1982-1994 (note that brightness and colours include the contribution of the faint companion HDE 269858p at $\sim 2^{\prime \prime}$ ).

In Fig. 5a the light curve for the $V_{\mathrm{J}}$ magnitudes is largely based on the y magnitudes $\left(y \equiv V_{\mathrm{J}}\right)$ of the LTPV project. Some values are based on $U B V$ and $V B L U W$ photometry. There is excellent agreement between the var- ious $V_{\mathrm{J}}$ scales. The uncertainty of each data point is of the order of \pm 0 . 01 .

The $(B-V)_{\mathrm{J}}$ curve is based on a few $U B V$ observations by de Groot (plusses, quoted by Stahl et al. 1983) and on the transformed $V-B$ of the Walraven system. Note that the magnitude scales for $(B-V)_{\mathrm{J}}, b-y$ and $v-b$ are twice those for $V_{\mathrm{J}}$ and $u-v$.

In Fig. 5b a smooth curve has been sketched through the data points (dotted when the time gap is too long) showing the fine structure of the rising branch, the broad maximum and the start of a descending branch.

\subsection{The fine structure and the photometric characteristics of the 1986-1993 maximum of $R$ 127}

Stahl et al. (1983) adopted a minimum brightness for $\mathrm{R} 127$ of $V_{\mathrm{J}} \sim 11.4$ (with a correction of 0.2 for the faint companion at a distance of $\sim 2^{\prime \prime}$ and with $V_{\mathrm{J}} \sim 13$ ) as observed by Mendoza (1970) in 1969. This is similar to the HDE photographic magnitude determined at the end of the 19th century, corrected by 0.3 to $V_{\mathrm{J}}$ by Feast et al. (1960). Three years prior to 1969, the brightness was higher by 0.8 : $V_{\mathrm{J}}=10.6$ (van Genderen 1970, corrected for the faint companion). Consequently, the observations presented in Fig. 5a lying between $V_{\mathrm{J}}=10.5$ (corrected for the faint companion) and 8.8 (companion negligible) represent an SD-type activity with a preceding minimum at JD $2444500\left(10^{\mathrm{m}} 5\right.$, see the insert), a rising branch with three $\sim 0.5$ amplitude cycles numbered $1-3$ in Fig. $5 \mathrm{a}$ each lasting $1.4 \mathrm{y}$, a broad maximum lasting $\sim 7 \mathrm{y}$, and a slow descent.

Figure 5a shows details of the three peaks $1-3$ during the early start of the rising branch. These SD phases show the expected colour variation, blue in the minima and red in the maxima. They are superimposed on the rise of what we would consider a VLT-SD cycle (dash-dotted curve in the insert). We suggest that maxima $1-3$ be considered normal SD phases with maximum 3 interrupted ("filled in") by the steep rise of the VLT-SD phase, like e.g. the maxima 36 and 37 of AG Car and the normal SD phases of S Dor (Paper I).

As far as we can judge the micro-variations superimposed on maxima $1-3$ tend to belong to the "100 d type" (although the precise time scale cannot always be established with certainty due to time gaps). Their colours are red in the maxima and blue in the minima. An exception may be the small jump at JD 2446060 which is "bright and blue". Although its descending branch seems to last as long as $50 \mathrm{~d}$, it is not certain that this peak should be classified as of the "100d type".

The broad 1986-1993 maximum (Figs. 5a,b) shows the typical characteristics of an SD phase like the 1990-1993 maximum of HR Car (Fig. 3): a strong reddening of the colours starting midway the rising branch of the $V_{\mathrm{J}}$ curve. The amplitudes of the light variations show a steep progressive decline to shorter wavelenghts: from 1.4 in $V_{\mathrm{J}}$ to 
$0^{\mathrm{m}} 5$ in $W_{\mathrm{w}}$. Maximum light is first reached at the shortest wavelengths and latest in the visual. The time difference between the maxima in $W_{\mathrm{w}}$ and $V_{\mathrm{J}}$ is at least $1000 \mathrm{~d}$. The same is true for S Dor (Paper I), but not for HR Car where light reaches maximum in all wavelenghts simultaneously (Fig. 3). At maximum $V_{\mathrm{J}}$, the $W_{\mathrm{w}}$ brightness of R 127 has already declined to roughly the same value as the minimum at JD 2445500. The same fast decline in the ultraviolet has been noticed for S Dor (Paper I).

This broad maximum shows at least a dozen peaks of micro-variations with amplitudes of $\sim 0{ }^{\mathrm{m}} 1$. Although it is difficult to determine all the individual time scales, the general impression is that they are of the order of $100 \mathrm{~d}$. In most cases colours are red in the maxima and blue in the minima. However, their behaviour is not always consistent: like in S Dor some peaks show a mixed colour behaviour or are consistently "bright and blue" (as at JD 2446450 and JD 2448650).

The detailed light and colour variations of a few typical micro-variations are shown in Fig. 6. While there are some short-time scale micro-variations in addition to the 100 d-type micro-variations, they are generally red in the maxima and blue in the minima.

It seems peculiar that the time scale of the $100 \mathrm{~d}$ type micro-variations of R 127 hardly changes during the brightness rise of $\sim 1 \mathrm{mag}$ in $V_{\mathrm{J}}$. At least, it does not change more than, say, $50 \%$ and there is no obviously continuous time-scale variation as a function of the star's brightness. This characteristic is shared with similar micro-variations of HR Car (Sect. 3.3), apparently creating a paradox if one wants to explain these microvariations as the result of stellar pulsations. After all, one would expect that with the apparent rise in brightness the radius increases and the density decreases so that the period of radial pulsations increases. In the case of R 127 a rough guess indicates that the stellar radius should increase by at least a factor of four from light minimum at JD $2444500\left(V_{\mathrm{J}}=10.5\right.$, note that the real minimum is likely $V_{\mathrm{J}}=11.4$, see above) to maximum, assuming that the expansion of the star is responsible for the total brightness rise.

Consequently, we have different possibilities. One of these is that the broad maximum is partly caused by an optically thick cool expanding shell making the star invisible. This shell could then be responsible for the $100 \mathrm{~d}$ variations caused by responding to the changing flux produced by the stellar oscillations. Consequently, the $100 \mathrm{~d}$ variations can then no longer be considered direct stellar pulsations. In this case the sometimes simultaneous presence of both short- and long-time scale micro-variations still needs an explanation. Another possibility is that in the expanding star the photospheric instability responsible for the short-time scale micro-variations dominant near minimum brightness is replaced by another type of photospheric instability that produces $100 \mathrm{~d}$ micro-variations which become the more dominant ones halfway up the ascending branch. In this case the non-continuous dependence of the time scale on the stellar density needs further explanation.

\section{R 40}

\subsection{The light and colour curves of $R$ 40, 1957-1994}

$\mathrm{R} 40=\mathrm{HD} 6884$ was discovered as an S Dor variable or LBV by Szeifert et al. (1993). The spectral type at minimum light around 1960 was B8Ie (Feast et al. 1960). Table 2 lists the global characteristics of the light and colour curves.

Figure 7 shows the light and colour curves of R 40 covering the time interval 1985-1994. The colour curves $b-y$, $v-b$ and $u-b$ (note: not $u-v$ ) are relative to the two comparison stars $\mathrm{A}$ and $\mathrm{B}$ in the sense variable minus $(\mathrm{A}+\mathrm{B}) / 2$. The error in brightness and colours is of the order of $\pm 0 .{ }^{\mathrm{m}} 01$.

The insert shows the schematic light curve $V_{\mathrm{J}}$ for the time interval 1957-1994. The dots are the scattered observations collected from the literature by Szeifert et al. (1993) and the smooth curve represents the underlying light curve from the large diagram. The data sets separated from each other by gaps in time are numbered $1-10$. Brightness and colours have the same magnitude scales.

Smooth curves have been sketched through the forest of schematically indicated micro-variations ( $V_{\mathrm{J}}$ amplitudes $0{ }^{\mathrm{m}} 1-0 \mathrm{~m} 2$; time scale $1-3$ months). Visual observations by A. Jones (for a description, see Sterken et al. 1996b) during the same time interval have a scatter of \pm 0.3 and globally show the same trend as the $V_{\mathrm{J}}$ curve. According to Jones' observations which continue until the end of 1995 (ours run only until the end of 1994), the brightness still rose another $0{ }^{\mathrm{m}} 1$. There is excellent agreement between the $V_{\mathrm{J}}$ of the LTPV project made with filter systems 6 and 7 and the $V_{\mathrm{J}}$ transformed from the Walraven $V$. However, there is a systematic difference between the latter and the observations made with filter system 8 of the LTPV project. This follows from observations made simultaneously on 13 nights. The correction applied to the filter 8 magnitudes equals $-0{ }^{\mathrm{m}} 042 \pm 0.012$.

The behaviour of brightness and colours from 1985 to 1994 is again typical for an SD phase: the colours become redder, especially in the $u-b$ colour index which reddens by $\sim 0{ }^{\mathrm{m}} 5$, while the rise in the visual was only $0{ }^{\mathrm{m}} 6$. Thus, it appears that by the time the $V_{\mathrm{J}}$ curve has reached the top of the ascending branch, the $u$ magnitude has dropped nearly to its minimum value. If this behaviour is typical for LBVs, as shown by S Dor, HR Car and R 127, it suggests that at the end of our ascending branch, R 40 is close to its visual maximum. 


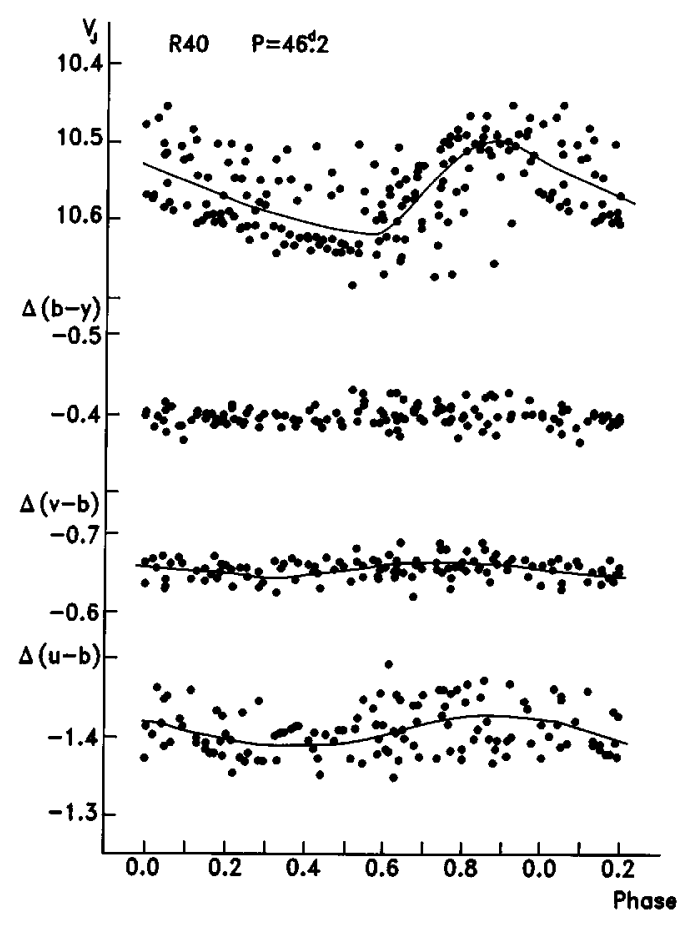

Fig. 8. The phase diagram for the light and colour curves of the micro-variations of $\mathrm{R} 40$ in data set $1-4$ for a period of 46.2 . The $V_{\mathrm{J}}$ curve also contains the results of the Walraven system; the colours are from the Strömgren system only

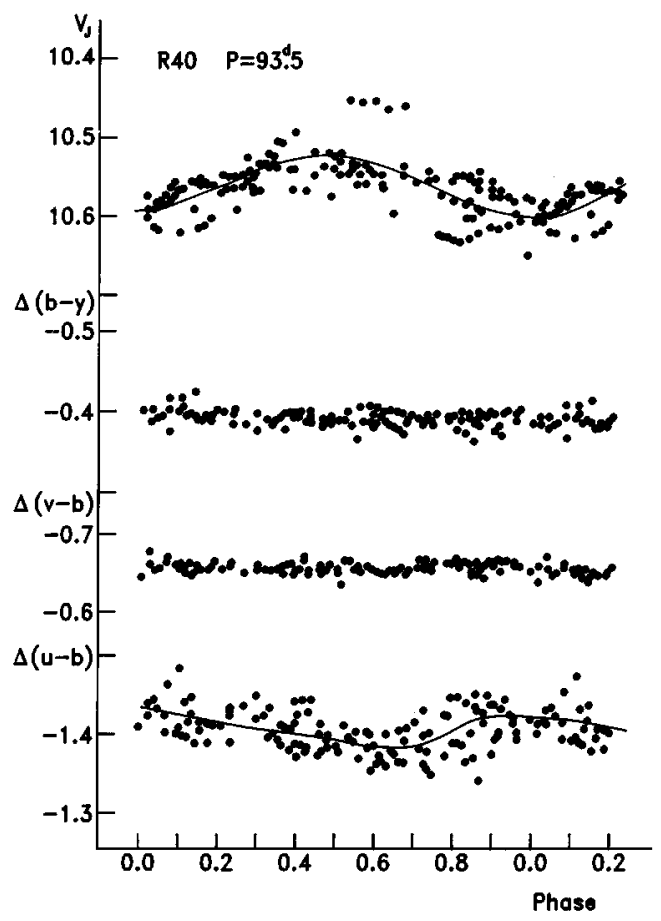

Fig. 9. The same as Fig. 8, but now for data set $5-7$ and a period of 93.5 after sets $1-4$ must be related to the change to another variability mechanism with a time scale of about $100 \mathrm{~d}$ as for the other LBVs investigated near maximum brightness.

2. The scatter around the mean curves, when larger than \pm 0.01 , is of intrinsic nature and is largest for $V_{\mathrm{J}}$. That is, there is a large variation in the light-curve shape from cycle to cycle. Evidently, a variety of time scales may be involved and more data might show complex periodicities.

The scatter in the $u-b$ curves is significantly larger than that in the $b-y$ and $v-b$ curves, pointing to additional fluctuations in the Balmer continuum and Balmer jump. We suspect that this may be caused by non-thermal photospheric velocity fields (like in the LBV HD 160529, studied by Wolf et al. 1974) and/or by atmospheric turbulence (van Genderen 1991). A large intrinsic scatter in colour indices which include an ultraviolet pass band has also been noticed in the behaviour of other $\alpha$ Cyg variables observed in the Walraven system (van Genderen 1991).

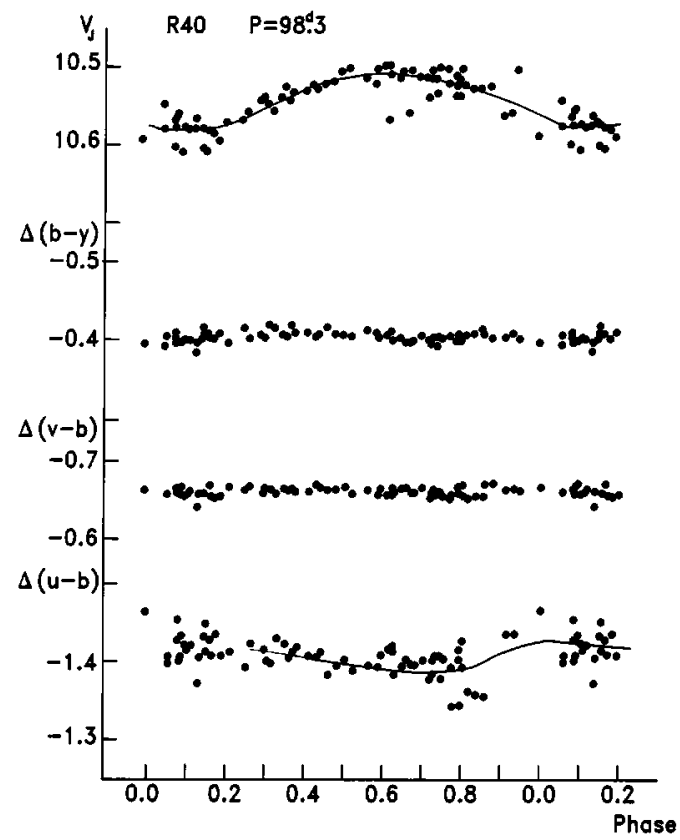

Fig. 10. The same as Fig. 8 , but now for data set $8-10$ and a period of 98.3

\section{6. $\mathrm{R} 71$}

6.1. The time scales of $S D$ phases and micro-variations of $R 71$

R $71=$ HDE 269006 had a spectral type B2.5Iep (Feast et al. 1960) at minimum brightness. It was already noted by Thackeray (1974) and Feast et al. (1960) that R 71 was 


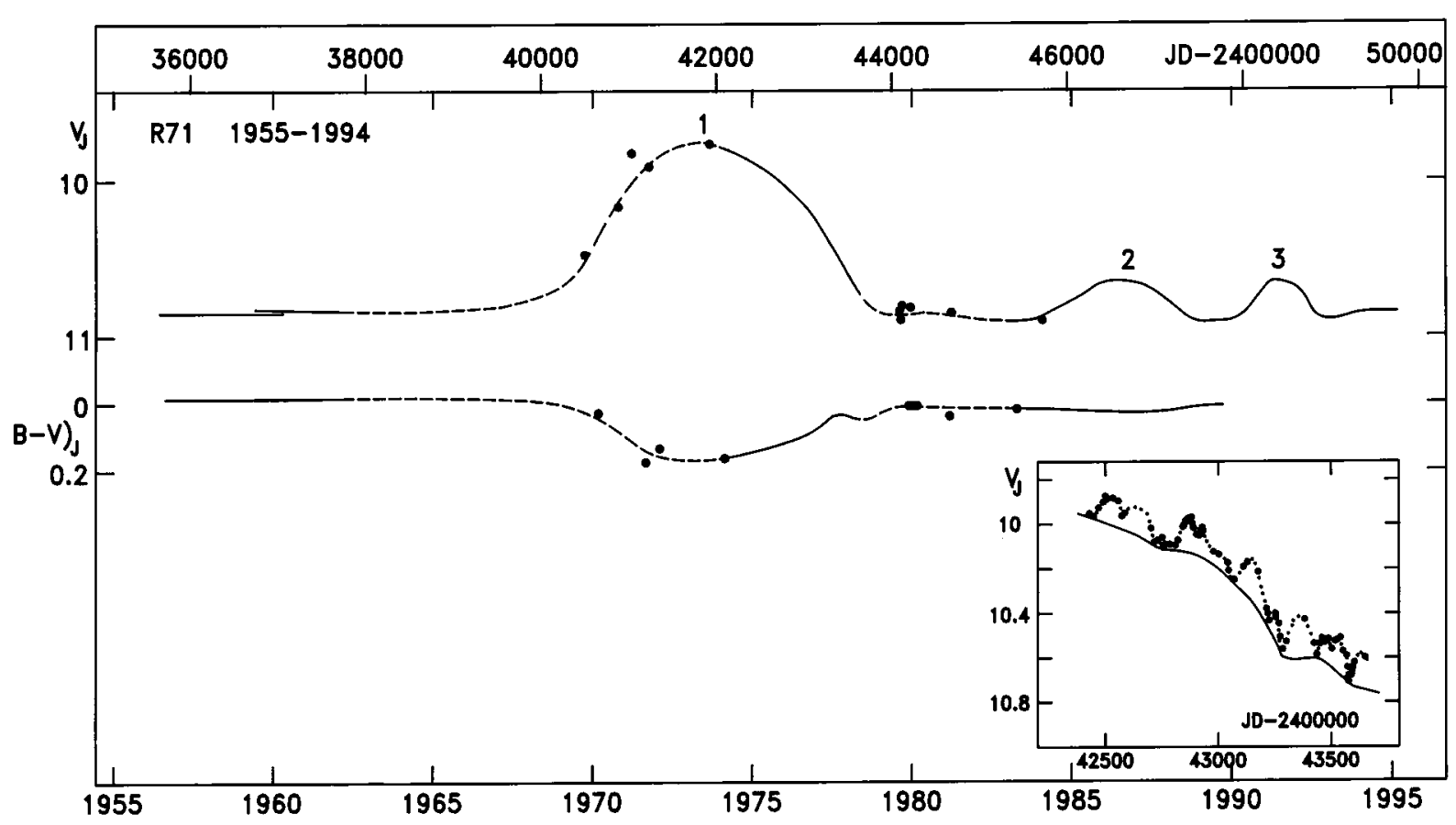

Fig. 11. The schematic photometric history of R 71, 1955-1994. The insert shows the detailed descending branch of maximum 1 with the long-time scale micro-variations

variable. The HDE magnitude at the end of the 19th century was 9.2 and the $m_{\mathrm{pg}}$ of the Cape Phot. Durchm. at roughly the same time was $9 \mathrm{~m} 5$. Additionally, according to Herschel's observations, $m_{v}=9.5$ in 1847 (Thackeray 1974). Thus in the middle of the last century, and also $50 \mathrm{y}$ later, R 71 was brighter than the top of the 1975 maximum shown in Fig. 11 (9m9). According to Thackeray (1974) the Harvard photographic magnitude indicated that R 71 was again bright in 1925 , viz. $9 \mathrm{~m}$. 2 . Thus, the total range in the last $150 \mathrm{y}$ is at least $1 \mathrm{~m} 8$. Table 2 lists the global characteristics of the light and colour variations during the last 40 y. Van Genderen (1979) discussed the photometric history of R 71 from 1957 to 1978 including the monitoring of an SD maximum and a subsequent descending branch.

Figure 11 shows at least two SD phases (numbered $1-2$ ) that are red in the maxima and blue in the minima. For maximum 3 no colour variations are known yet. The two straight line pieces in the very beginning represent two individual observations of which the precise dates are unknown. Scattered individual observations are indicated by dots. Monitored parts are indicated by continuous curves. Numerous fluctuations in the descending branch are shown in the insert. According to their time scale $(100 \mathrm{~d}-200 \mathrm{~d})$ and colour behaviour these should be classified as long-time scale micro-variations. After the fading of S Dor phase No. 1 short-time scale micro-variations with quasi-periods of $24 \mathrm{~d}$ and $14 \mathrm{~d}$ were recorded (van Genderen et al. 1985, 1988). New unpublished $V B L U W$ observations made in 1988 and 1989 (Sect. 2) show a quasi- period of $20 \mathrm{~d}$. All these short-time scale variations are generally blue in the maxima and red in the minima.

The $(B-V)_{\mathrm{J}}$ curve in Fig. 11 is based on observations made in the Johnson $U B V$ system and on transformed $V-B$ values of the Walraven system. Spectroscopic monitoring done between 1955 and 1981 offers a check on whether R 71 was active or not in this time interval: in the light maxima the emission lines of [Fe II] are absent and in the light minima they are present (Thackeray 1974; Wolf et al. 1981; see the corresponding dates in the light curve of van Genderen et al. 1985). According to spectra taken between 1955 and 1965, there was no SD type activity whatsoever. Consequently, we are pretty sure that R 71 was in a minimum state all that time (see the broken curve 1960-1970 in Fig. 11).

\section{R 110}

\subsection{The light and colour curves of $R 110,1957-1994$}

R $110=$ HDE 269662 was discovered as an LBV by Stahl et al. (1990), but was suspected of photometric variability already in 1984 (Stahl et al. 1984). Table 2 lists the global characteristics of light and colour curves during the last $40 \mathrm{y}$.

Figure 12 shows the recent photometric history of R 110 from the first photoelectric observations around 1957 up to 1994, in various photometric systems. All scattered observations are represented by dots in the main diagram for $V_{\mathrm{J}}$ and in the panel for $(B-V)_{\mathrm{J}}$. All 
variables the wind characteristics and the pseudophotosphere (e.g. Stahl et al. 1990) may play an increasing role in the photometric behaviour. Whether R 110 can be classified as an LYV cannot be answered before a reliable temperature determination during visual maximum has been made.

\section{Discussion and conclusions}

Together with the results of Paper I, we now have an outline of the variability of $8 \mathrm{LBVs}$ over a time interval of the order of a century or more. In four cases the SD activity is reasonably well-covered by photometric observations over $50-150$ y: AG Car, S Dor, $\eta$ Car (Paper I) and HR Car (present paper Sect. 3). The time scales for the VLT-SD phases lie in the order of $20-50 y$. For the four other cases, a few scattered observations were made in the past with long time gaps in between, while monitoring programs were carried out only within the last few decades: R 40 (SMC), R 71, R 110 and R 127 (LMC). Thus, for these stars only details of the normal SD phases and the superimposed micro-variations could be documented. The pronounced individuality of these stars, especially with respect to the SD phases, is most striking. We summarize the characteristics of the micro-variations of the LBVs as follows:

Near minimum light all LBVs investigated so far show micro-variations (amplitudes $\sim 0.2$ ) on a time scale of $15-45 \mathrm{~d}$ (called $\alpha$ Cyg variations in Paper I). The lightcurve characteristics change from cycle to cycle as for normal $\alpha$ Cyg variables and quasi-periods may differ significantly at different epochs. This could mean that multimode pulsations are present. Detailed analyses of the light variations of $\eta$ Car and $\zeta^{1}$ Sco offer strong additional evidence for this statement (Sterken et al. 1996a, 1997). These micro-variations are generally blue in the maxima and red in the minima.

Near maximum light LBVs show micro-variations (amplitudes also $\sim 0.2$ ) on a longer time scale, viz. $\sim 100 \mathrm{~d}$. These are generally red in the maxima and blue in the minima. It is a pity that we could not establish whether AG Car's maxima showed the same type of microvariation because of the lack of sufficient photoelectric monitoring.

The change of the short-time scale micro-variations to the long-time scale ones and vice versa happens rather abruptly, within at most a few hundred days, somewhere along the rising and declining branches at some temperature between $10000 \mathrm{~K}$ and $15000 \mathrm{~K}$. The corresponding change in the time scale is relatively small (e.g. a factor two for R 40), while one would expect this factor to be much larger if the brightness variation is due only to the expansion of the star (i.e. a decreasing density) and if both types of micro-variations are due to radial pulsations. Apart from that, the $\sim 100 \mathrm{~d}$ micro-variations do not vary much in time scale as the steller brightness continues to increase (e.g., by $0^{\mathrm{m}} 5$ for $\mathrm{HR}$ Car and by $\sim 1$ mag for R 127). Obviously, short- and long-time scale micro-variations are caused by different instability mechanisms. The long-time scale variations could be caused either by a thick shell responding to possible stellar pulsations, or by the expanded stellar photosphere being subject to a new type of photospheric instability.

In this context it is very relevant to mention the spectroscopic studies by Wolf (1972) and Hillendahl (1970) of HD 33579 (non-LBV), by Leitherer et al. (1985) and by Wolf \& Stahl (1990) of S Dor in maximum and by Wolf (1992) of S Dor and R 127 in maximum. They analyzed emission-line variations pointing to pulsation-like movements and depth-dependent velocity fields on a time scale of months. At the dates denoted by arrows in Fig. 5a (R 127), Wolf (1992) has obtained spectra between 4450 and $4560 \AA$. Sometimes these stars exhibit inverse P Cygtype line profiles pointing to a very unstable mass outflow, with large clumps of matter falling back to the star. In this case kinetic energy can be released. Attempts have been made to link these spectroscopic changes to the observed photometric micro-variations, thus far with little success. Only in a recent study of the LBV P Cyg, some evidence was found for changes in the brightness of the star at the epochs of shell ejections (Israelian et al. 1996).

Large turbulent elements and dynamical instabilities in super- and hypergiant photospheres exist according to the studies of e.g. de Jager et al. (1991), Nieuwenhuijzen \& de Jager (1995) and Nieuwenhuijzen et al. (1997).

It is not unlikely that all these phenomena, partly or largely of a stochastic nature, contribute to the long-time scale micro-variations and their sometimes intricate colour behaviour. These problems can only be solved by simultaneous spectroscopic and photometric monitoring campaigns for which medium-sized and small telescopes, respectively, are most useful.

It should be recalled that the above-mentioned Atype hypergiant HD 33579 shows long-time scale microvariations like the LBVs in maximum and with a quasi-period of $\sim 100 \mathrm{~d}$, generally, however, with a reversed colour behaviour, i.e. blue in the maxima and red in the minima (van Genderen 1979). According to Nieuwenhuijzen et al. (1997) this star is a redward evolving star showing an atmosphere permeated by shock waves covering a large fraction of the visible surface and following each other in time intervals of some $100 \mathrm{~d}$. This supports the multi-colour photometric evidence that the 100 d-type variations of HD 33579 cannot be explained by pulsations (van Genderen 1979). However, the preference for the reversed colour behaviour compared to that of the LBVs is very puzzling. Evidently, normal A/F-type $\alpha$ Cyg variables and LBVs in maximum, although having the same temperatures, behave not quite similar.

It is no exaggeration to state that LBVs can be considered as the most complicated stars among the pulsating variables. They form an excellent and well-equipped 
Table 2. Global characteristics of the best observed parts of the light and colour curves. Time scales for the SD phases are given in years, those for the micro-variations in days

\begin{tabular}{|c|c|c|c|c|c|c|}
\hline \multirow[t]{2}{*}{ Star } & \multirow{2}{*}{$\begin{array}{c}\text { JD-2400000 } \\
\text { (Date) }\end{array}$} & \multirow[t]{2}{*}{ Features } & \multirow[t]{2}{*}{ Time scale } & \multirow{2}{*}{$\begin{array}{l}\text { Ampl. } \\
\text { (mag) }\end{array}$} & \multicolumn{2}{|c|}{ colour in } \\
\hline & & & & & $\max$ & $\min$ \\
\hline \multirow[t]{4}{*}{ HR Car } & $\begin{array}{l}13000-30000 \\
(1889-1938)\end{array}$ & 3 maxima & $5.5-8 \mathrm{y}$ & $1-1.5$ & & \\
\hline & $\begin{array}{l}45000-49000 \\
(1982-1994)\end{array}$ & 3 maxima & $2-2.7 \mathrm{y}$ & 0.5 & red & blue \\
\hline & & $\begin{array}{l}\text { micro var. } \\
\text { near min } \\
\text { micro var. }\end{array}$ & $20-40 \mathrm{~d}$ & 0.15 & blue & red \\
\hline & & near $\max$ & $100 \mathrm{~d}$ & 0.2 & red & blue \\
\hline \multirow[t]{3}{*}{ R 127} & $\begin{array}{l}44500-49500 \\
(1981-1994)\end{array}$ & 3 maxima & $1.4 \mathrm{y}$ & 0.5 & red & blue \\
\hline & & $\begin{array}{l}1 \text { maximum } \\
\text { micro var. }\end{array}$ & $7 y$ & 1.7 & red & blue \\
\hline & & near max & $100 \mathrm{~d}$ & 0.1 & red & blue \\
\hline \multirow[t]{4}{*}{ R 40} & $\begin{array}{l}36000-49500 \\
(1957-1994)\end{array}$ & 1 maximum & $25 \mathrm{y}$ & 0.2 & - & - \\
\hline & & $\begin{array}{l}\text { rising } \\
\text { branch } \\
\text { micro var. }\end{array}$ & $7 y$ & 0.6 & red & blue \\
\hline & & $\begin{array}{l}\text { near min. } \\
\text { micro var. }\end{array}$ & $46 \mathrm{~d}$ & 0.1 & blue & red \\
\hline & & near max. & $100 \mathrm{~d}$ & 0.05 & red & blue \\
\hline \multirow[t]{4}{*}{ R 71} & $36000-49500$ & 3 maxima & $10 y$ & 1 & red & blue \\
\hline & $(1957-1994)$ & & $6 \mathrm{y}$ & 0.25 & red & blue \\
\hline & & & $3 y$ & 0.25 & - & - \\
\hline & & $\begin{array}{l}\text { micro var. } \\
\text { near min. } \\
\text { micro var. }\end{array}$ & $14-24 \mathrm{~d}$ & 0.1 & blue & red \\
\hline R 110 & $\begin{array}{l}36000-49500 \\
(1957-1994)\end{array}$ & $\begin{array}{l}\text { near max. } \\
3 \text { maxima }\end{array}$ & $\begin{array}{l}100-200 \mathrm{~d} \\
5-10 \mathrm{y}\end{array}$ & $\begin{array}{l}0.2 \\
0.3-0.6\end{array}$ & $\begin{array}{l}\text { red } \\
\text { red }\end{array}$ & $\begin{array}{l}\text { blue } \\
\text { blue }\end{array}$ \\
\hline
\end{tabular}

laboratory for the study of the different kinds of photospheric and atmospheric pulsations and instabilities of a massive star within a large range of temperature, radius and mean density.

Acknowledgements. CS acknowledges a research grant from the Belgian Fund for Scientific Research (NFWO). Research at the Armagh Observatory is grant-aided by the Department of Education for Northern Ireland, and by the UK PPARC through the provision of the Starlink network. The authors are indebted to the Association Française des Observateurs d'Etoiles Variables (AFOEV) for making public their magnitude estimates of HR Car. Part of the data were obtained during observing time allottments 55D-0317, 56D-0249 and 57D-0133. We are much indebted to the referee Dr. M.W. Feast for his critical evaluation.

\section{References}

Bateson F.M., 1987-1990, Circ. Roy. Astron. Soc. New Zealand Bond H.E., Landolt A.U., 1970, PASP 82, 313
Feast M.W., Thackaray A.D., Wesselink A.J., 1960, MNRAS 121,337

Gallagher J.S., 1992, in Nonisotropic and Variable Outflows from Stars, Drissen L., Leitherer C., Nota A. (eds.) ASP Conf. Ser. 22, 339

van Genderen A.M., 1970, A\&A 7, 49

van Genderen A.M., 1979, A\&AS 38, 381

van Genderen A.M., 1991, in ESO Workshop on Rapid Variability of OB-Stars: Nature and Diagnostic Value, Baade D. (ed.). Garching, p. 117

van Genderen A.M., van Leeuwen F., Brand J., 1982, A\&AS 47, 591

van Genderen A.M., Steemers W.J.G., Feldbrugge P.T.M., et al., 1985, A\&A 153, 163

van Genderen A.M., Thé P.S., Augusteijn Th., et al., 1988, A\&AS 74, 453

van Genderen A.M., Thé P.S., Heemskerk M., et al., 1990, A\&AS 82, 189

van Genderen A.M., Sterken C., de Groot M., 1996, A\&A (in press) (Paper I)

Graham J.A., 1968, BAN Suppl. 2, 397 
de Groot M., van Genderen A.M., Sterken C., 1996, Irish AJ $23(2), 187$

Hillendahl R.W., 1970, Symp. on Spectrum Formation in Stars with Steady-State Extended Atmospheres, NSB-Special Publ. 332-300

Hoffleit D., 1940, Harv.Bull. 913, 4

Hutsemékers D., van Drom E., 1991, A\&A 248, 141

Israelian G., de Groot M., Parker J.Wm., et al., 1996, A\&A (in press)

Isserstedt, J., 1975, A\&AS 19, 259

de Jager C., van Genderen A.M., 1989, in IAU Coll. 113, Physics of Luminous Blue Variables, Davidson K., Moffat A.F.J., Lamers H.J.L.G.M. (eds.). Kluwer, p. 127

de Jager C., de Koter A., Carpay J., et al., 1991, A\&A 244, 131

Kilkenny D., Whittet D.C.B., Davies J.K., et al., 1985, SAAO Circ. 9, 55

de Koter A., Lamers H.J.G.L.M., Schmutz W., 1996, A\&A 306, 501

Lamers H.J.G.L.M., 1995, in IAU Coll. 155, Astrophysical Applications of Stellar Pulsations, ASP Conf. Ser. 83, Stobie R.S., Whitelock P.A. (eds.) p. 176

Leitherer C., Appenzeller I., Klare G., et al., 1985, A\&A 153, 168

Manfroid J., Sterken C., Bruch A., et al., 1991, First Cat. Stars Measured in the LTPV Project (1982-1986) A\&AS 87, 481

Manfroid J., Sterken C., Cunow B., et al., 1994, Third Cat. Stars Measured in the LTPV Project (1990-1992) A\&AS 109, 329

Mendoza V.E.E., 1970, Bol. Obs. Tonantzintla y Tacubaya 5, 269

Nieuwenhuijzen H., de Jager C., 1995, A\&A 302, 811

Nieuwenhuijzen H., de Jager C., Groth H.G., 1997, A\&A (submitted)

Shore S.N., Brown D.N., Bopp B.W., et al., 1990, ApJS 73, 461

Spoon H.W.W., de Koter A., Sterken C., et al., 1994, A\&AS 106, 141
Stahl O., Wolf B., Klare G., et al., 1983, A\&A 127, 49

Stahl O., Wolf B., Leitherer C., et al., 1984, A\&A 140, 459

Stahl O., Wolf B., 1986, A\&A 154, 243

Stahl O., Wolf B., Klare G., et al., 1990, A\&A 228, 379

Sterken C., 1977, A\&A 57, 361

Sterken C., 1981, in ESO Workshop: The Most Massive Stars, D'Odorico S., Baade D., Kjär K. (eds.) p. 147

Sterken C., 1983, The Messenger 33, 10

Sterken C., Gosset E., Jüttner A., et al., 1991, A\&A 247, 383

Sterken C., Manfroid J., Anton K., et al., 1993, Second Cat. Stars Measured in the LTPV Project (1986-1990) A\&AS 102,79

Sterken C., Manfroid J., Beele D., et al., 1995, Fourth Cat. Stars Measured in the LTPV Project (1992-1994) A\&AS 113,31

Sterken C., de Groot M., van Genderen A.M., 1996a, A\&AS 116,9

Sterken C., Jones, A., van Genderen A.M., de Groot M., 1996b, in: Astronomical photometry into the next millenium, Proc. P.E.P. 5, B. Carter \& J. O'Mara (eds.) (in press)

Sterken C., de Groot M., van Genderen A.M., 1997, A\&A (in press)

Szeifert Th., Stahl O., Wolf B., et al., 1993, A\&A 280, 508

Thackeray A.D., 1974, MNRAS 168, 221

Walborn N.R., 1977, ApJ 215, 53

Walborn N.R., 1982, ApJ 256, 452

Walraven Th., Walraven J.H., 1977, A\&AS 30, 245

Wisse M., Wisse P.J.N., 1971, A\&A 12, 149

Wolf B., 1972, A\&A 20, 275

Wolf B., 1989, in Phyisics of Luminous Blue Variables, IAU Coll. 113, Davidson K., Moffat A.F.J., Lamers H.J.G.L.M. (eds.). Kluwer, p. 96

Wolf B., 1992, in Nonisotropic Outflows from Stars, Drissen L., Leitherer C., Nota A. (eds.). ASP Conf. Ser. 22, 327

Wolf B., Campusano L., Sterken C., 1974, A\&A 36, 87

Wolf B., Appenzeller I., Stahl O., 1981, A\&A 103, 94

Wolf B., Stahl O., 1990, A\&A 235, 340 